\title{
Multiwavelength observations of a flux rope formation by series of magnetic reconnection in the chromosphere ${ }^{\star}$
}

\author{
Pankaj Kumar ${ }^{1,2}$, Vasyl Yurchyshyn ${ }^{3,1}$, Kyung-Suk Cho ${ }^{1,5}$, and Haimin Wang ${ }^{4,3}$ \\ 1 Korea Astronomy and Space Science Institute (KASI), 305-348 Daejeon, Republic of Korea \\ e-mail: pankaj@kasi.re.kr \\ 2 Heliophysics Science Division, NASA Goddard Space Flight Center, Greenbelt, MD 20771, USA \\ 3 Big Bear Solar Observatory, New Jersey Institute of Technology, Big Bear City, CA 92314, USA \\ ${ }_{5}^{4}$ Space Weather Research Laboratory, New Jersey Institute of Technology, University Heights, Newark, NJ 07102-1982, USA \\ 5 University of Science and Technology, 305-348 Daejeon, Republic of Korea
}

Received 12 July 2016 / Accepted 28 March 2017

\begin{abstract}
Using high-resolution observations from the $1.6 \mathrm{~m}$ New Solar Telescope (NST) operating at the Big Bear Solar Observatory (BBSO), we report direct evidence of merging and reconnection of cool $\mathrm{H} \alpha$ loops in the chromosphere during two homologous flares (B and $\mathrm{C}$ class) caused by a shear motion at the footpoints of two loops. The reconnection between these loops caused the formation of an unstable flux rope that showed counterclockwise rotation. The flux rope could not reach the height of torus instability and failed to form a coronal mass ejection. The HMI magnetograms revealed rotation of the negative and positive (N1/P2) polarity sunspots in the opposite directions, which increased the right- and left-handed twist in the magnetic structures rooted at N1/P2. Rapid photospheric flux cancellation (duration $\sim 20-30 \mathrm{~min}$, rate $\approx 3.44 \times 10^{20} \mathrm{Mx} \mathrm{h}^{-1}$ ) was observed during and even after the first B6.0 flare and continued until the end of the second C2.3 flare. The RHESSI X-ray sources were located at the site of the loop coalescence. To the best of our knowledge, such a clear interaction of chromospheric loops along with rapid flux cancellation has not been reported before. These high-resolution observations suggest the formation of a small flux rope by a series of magnetic reconnections within chromospheric loops that are associated with very rapid flux cancellation.
\end{abstract}

Key words. Sun: chromosphere - Sun: magnetic fields - Sun: flares - Sun: coronal mass ejections (CMEs) - Sun: oscillations Sun: particle emission

\section{Introduction}

Flux ropes contain helically twisted field lines wrapped around a central axis and are considered an integral part of a coronal mass ejection (CME, Rust \& Kumar 1996; Chen 2011). The flux ropes are recognized as a magnetic cloud in the interplanetary medium that generally exhibit strong magnetic field, low plasma beta, and smooth rotation of the magnetic field vector $\left(B_{\theta}\right)$ at 1 AU (Burlaga et al. 1982; Marubashi 1986; Bothmer \& Schwenn 1998; Yurchyshyn et al. 2001). These flux ropes (with a strong southward magnetic field component) can produce severe geomagnetic storms by interacting with Earth's magnetosphere. Therefore, the study of the flux rope formation and eruption is important for the space weather research (Srivastava \& Venkatakrishnan 2004; Gopalswamy et al. 2005; Kumar et al. 2011a).

The formation of erupting flux ropes on the Sun is not very well understood. The presence of a flux rope before or during an eruption is a crucial condition for CME initiation models. Some CME initiation models (e.g., emerging flux model: Chen \& Shibata 2000, magnetohydrodynamic kink instability models: Fan \& Gibson 2004; Kliem et al. 2004; Török \& Kliem 2005) assume a preexisting twisted flux rope before the eruption, whereas in other models (e.g., tether-cutting model: Moore et al. 2001, breakout models: Antiochos 1998; Karpen et al. 2012) the

\footnotetext{
* Movies attached to Figs. 2, 7, 8, and 10 are available at http: //www . aanda.org
}

flux rope is formed (during the eruption) in the corona by a series of magnetic reconnections within an arcade of loops. The forward-S- or inverse-S-shaped sigmoids are generally observed in the X-ray images of active regions (ARs), which may be the manifestation of kink-unstable flux ropes (Canfield et al. 1999; Rust \& Kumar 1996; Kliem et al. 2004; Gibson et al. 2006). According to the flux rope model of the prominence or filament (van Ballegooijen \& Martens 1989), a series of magnetic reconnections between the sheared arcade of loops at the polarity inversion line (PIL) driven by photospheric converging motions leads to the formation of a helical flux rope before it erupts. Other models create a flux rope during the eruption process using multiple reconnections of the arcade loops during a two-ribbon flare that leaves behind a post-eruptive arcade (Gosling et al. 1995; Longcope \& Beveridge 2007). An alternative assumption is that the flux rope emerges from below the photosphere and is already twisted in the convection zone (Rust \& Kumar 1994; McKaig 2001).

The three-part structure of a CME includes a frontal loop or leading edge, a dark cavity, and a bright core region (filament or prominence material). The cavity region of a CME is assumed to be a flux rope (Bạk-Stẹślicka et al. 2013). According to the flux rope model (Priest et al. 1989) of a solar prominence, the filament cool material is supported in the lower portion of the helical field lines. The circular features within the CMEs observed by coronagraphs are considered to be a helical flux rope structure (Vourlidas et al. 2013; Vourlidas 2014). The 
interplanetary scintillation images (density) also show the evolution of the large-scale CME flux ropes in the interplanetary medium (50-250 Rs) (Manoharan 2010).

There are a number of observational reports on the activation of a helical flux rope that is associated with kink instability. For example, helical field lines with three to four turns have been reported during a kink-unstable prominence eruption (Gary \& Moore 2004; Kumar et al. 2012). In addition, using multiwavelength observations (Hinode/SOT, TRACE), Srivastava et al. (2010) and Kumar et al. (2010) provided evidence of a helically kinked flux tube (three to four turns) that triggered multiple B- and M-class flares associated with a failed eruption. Kumar et al. (2011b) reported a failed flux rope eruption observed not only in the STEREO and TRACE EUV cool channels, but also in the XRT hot channel, which suggests that multi-temperature plasma may be contained within a flux rope. Recently, SDO/AIA observations revealed high-temperature flux ropes visible in 131 and $94 \AA$ channels (Cheng et al. 2011; Zhang et al. 2012; Kumar \& Innes 2013). However, the above reports did not discuss the formation mechanism of the flux ropes, mostly because they lacked photospheric magnetograms and/or high-resolution chromospheric observations.

Using high-resolution Hinode/SOT images, Okamoto et al (2008) presented evidence for the emergence of a helical flux rope from below a preexisting prominence. Green \& Kliem (2009), Tripathi et al. (2009), and Green et al. (2011) suggested the formation of an S-shaped flux rope through flux cancellation at the PIL. Liu et al. (2010) showed evidence of tethercutting reconnection between two opposite J-shaped coronal loops that formed a continuous S-shaped flux rope. Recently, Kumar \& Cho (2014) reported reconnection signatures above a small kinked filament and formation of a twisted hot flux rope observed in the AIA 131, $94 \AA$, and XRT images during magnetic reconnection. These observations show the formation of coronal flux ropes.

Several mechanisms are capable of triggering and driving the flux rope eruption. The flux rope eruption can be triggered either by a reconnection process (e.g., associated with tether cutting or an emerging flux) or by a magnetohydrodynamic (MHD) instability (i.e., kink instability if the twist angle $\geq 2.49 \pi$, Hood \& Priest 1981; Einaudi \& van Hoven 1983), which may cause it to attain a certain height within an AR. Furthermore, the successful eruption largely depends on the onset of the torus instability (Kliem \& Török 2006; Olmedo \& Zhang 2010; Aulanier et al. 2010), which is defined by the rate of decrease in the overlying magnetic field with height (i.e., decay index $\geq 1.5$ Kliem \& Török 2006).

The high-resolution observations from the NST are extremely useful to investigate the issues related to the flux rope formation and eruption from the photosphere to chromosphere. A recent study using NST data along with an NLFFF modeling revealed a preexisting small flux rope in the $\mathrm{H} \alpha$ chromosphere that became unstable most likely because of the onset of a kink instability (Wang et al. 2015). Alternatively, Kumar et al. (2015) provided evidence of reconnection (inflows) between cool chromospheric sheared loops and the associated appearance and formation of an S-shaped twisted flux rope that presumably was a result of magnetic reconnection. In addition, Yurchyshyn et al. (2015) reported an in situ formation of a flux rope by multiple reconnections starting from the low chromosphere to the corona. The flux rope was observed as a circular shape (axial view) in the hot AIA channels (131 and $94 \AA$ ) and LASCO C2 coronagraph images.
In this paper, we mainly focus on the interaction and reconnection between $\mathrm{H} \alpha$ loops, the flux rope formation, and its dynamics in the chromosphere and corona during two small homologous flares (B6.0 and C2.3) that occurred in AR NOAA 12353 on 23 May 2015. During both flares, we detected oscillatory reconnection in X-ray and EUV channels. A small flux rope was observed in high-resolution NST $\mathrm{H} \alpha$ images, allowing us to resolve the dynamics of small-scale chromospheric loops that are otherwise not detectable in the AIA data. We used SDO/AIA images and HMI magnetograms to investigate the structure and dynamics of the large-scale coronal magnetic field configuration (e.g., loop connectivity) and the associated photospheric field changes before and during the flare. RHESSI hard X-ray images are used to locate the particle transportation and precipitation site during the flares. In Sect. 2 we present the observations and the results. In the last section, we summarize and discuss our findings.

\section{Observation and results}

The NST images used in this study were obtained with the help of the 308 subaperture adaptive optics (AO-308) system. We used a series of narrow-band $\mathrm{H} \alpha(6563 \AA)$ images taken at $\pm 0.8 \AA, \pm 0.4 \AA$, and $0.0 \AA$ from the line center with the NST Visible Imaging Spectrometer (VIS, pixel size of $0.029^{\prime \prime}$ ). The VIS combines a $5 \AA$ interference filter with a Fabry-Pérot etalon to produce a resulting bandpass of $0.07 \AA$ over a $70^{\prime \prime} \times 70^{\prime \prime}$ field of view. A series of broadband (10 $\AA$ ) images of the photosphere were acquired with a TiO filter $\left(7057 \AA\right.$, pixel scale of $\left.0.0375^{\prime \prime}\right)$ to study the evolution of the fine photospheric structures associated with flux emergence and cancellation.

The Atmospheric Image Assembly (AIA; Lemen et al. 2012) onboard the Solar Dynamics Observatory (SDO; Pesnell et al. 2012) acquires full-disk images of the Sun (field of view $\left.\sim 1.3 R_{\odot}\right)$ with a spatial resolution of $1.5^{\prime \prime}\left(0.6^{\prime \prime}\right.$ pixel $\left.^{-1}\right)$ and a cadence of $12 \mathrm{~s}$ in ten extreme ultraviolet (EUV) and UV channels. This study uses $171 \AA$ Fe IX, $T \approx 0.7 \mathrm{MK}$ ), $131 \AA$ (Fe VIII, Fe XXI, Fe XXIII, i.e., 0.4, 10, 16 MK), $304 \AA$ (He II, $T \approx$ $0.05 \mathrm{MK})$, and $1600 \AA$ (C IV + continuum, $T \approx 0.1 \mathrm{MK} \&$ $5000 \mathrm{~K}$ ) images. We also used Heliospheric and Magnetic Imager (HMI) magnetograms (Schou et al. 2012) to investigate the magnetic field evolution before and during the flares.

We also used Reuven Ramaty High Energy Solar Spectroscopic Imager (RHESSI; Lin et al. 2002) data to investigate the particle acceleration and precipitation sites during the flares. The CLEAN algorithm was used for the image reconstruction with an integration time of $30 \mathrm{~s}$.

Active region NOAA 12353 of $\beta \gamma$ magnetic configuration was located near the disk center (N07W17) on 23 May 2015. We focus on two homologous flares that occurred at the same site within the AR and on the associated reconnection events that formed a twisted flux rope. Figure 1a displays the GOES soft X-ray flux profile obtained in the $1-8 \AA$ channel. The bottom panel of Fig. $1 \mathrm{~b}$ shows RHESSI X-ray flux in the $6-12 \mathrm{keV}$ (black) and 12-25 keV (blue) channels. The RHESSI did not observe the third peak of the second flare, therefore we overplotted Fermi GBM (Gamma Ray Burst Monitor; Meegan et al. 2009) X-ray flux profile (6-12 keV, red) in the same panel. The first B6.0 flare started at $\sim 16: 50 \mathrm{UT}$, peaked at $\sim 16: 53 \mathrm{UT}$, and ended at $\sim 17: 10 \mathrm{UT}$. We note that another short-duration B-class flare was detected at 16:50-16:55 UT that occurred in AR NOAA 12349 (S21W34). This flare contaminated the first peak of the B6.0 X-ray burst. The best way to exclude the 


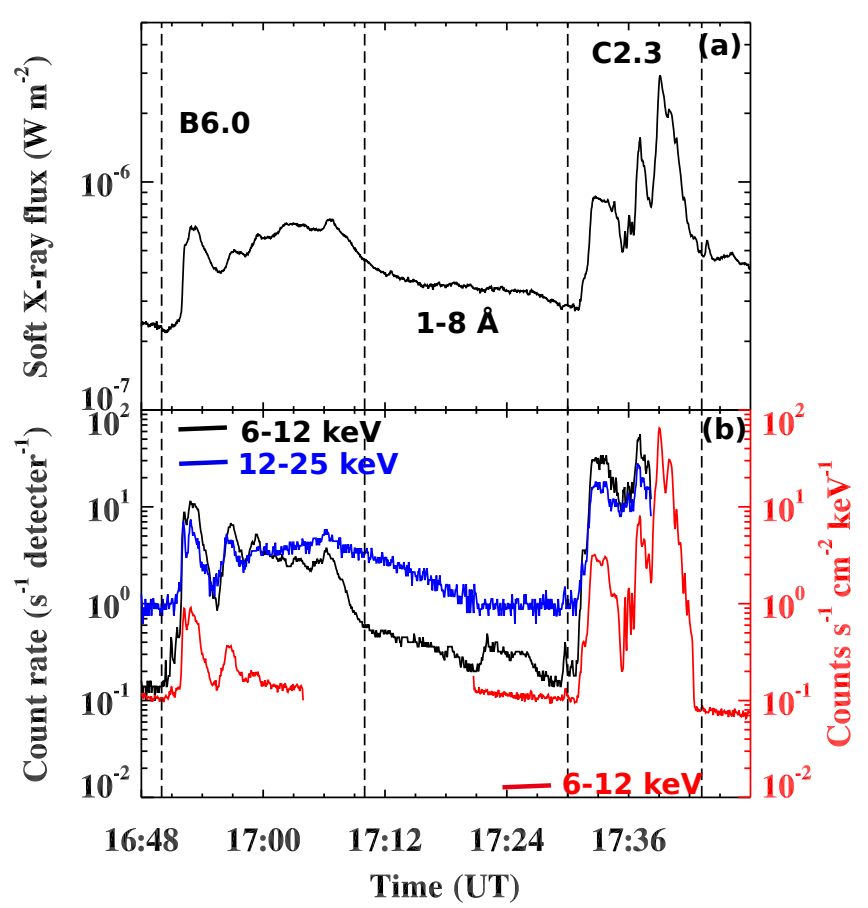

Fig. 1. a) GOES soft $X$-ray flux profile in $1-8 \AA$ channel. b) RHESSI $\mathrm{X}$-ray flux in the 6-12 keV (black) and 12-25 (blue) keV channels. The Fermi GBM X-ray flux in the 6-12 keV (red) is also included. The vertical dashed lines indicate the interval of the B6.0 and C2.3 flare.

contribution from the other AR is to plot the EUV flux profile of the studied AR in order to show the periodic behavior of the first B6.0 flare (see next section). The second C2.3 flare started at $\sim 17: 30$ UT, peaked at $\sim 17: 39$ UT, and ended at $\sim 17: 45$ UT. Interestingly, in both soft (6-12 keV) and hard (12-25 keV) X-ray time profiles we detected oscillatory behavior during the two flares.

\subsection{First B6.0 flare}

Figures 2a,b displays the AIA 171 and $304 \AA$ images of the AR overplotted with the HMI magnetogram contours of positive (white) and negative (black) polarities. In the AIA $171 \AA$ image, a set of overlying coronal loops (above the flare site) connects opposite polarities of the AR. The AIA $304 \AA$ image shows the connectivity of the dark and cool chromospheric or transition region loops (indicated by arrows). We do not see any preexisting filament lying along the PIL. Therefore the dark structures are mentioned as a system of cool loops connecting opposite polarity fields. Cool loops are also seen at this location in the high-resolution NST H $\alpha$ images (Fig. 6). Figures 2c-h shows the zoomed-in area outlined by the rectangular box in the panel a) as it appears in the AIA 171, 1600, 304, and $131 \AA$ channels. The initial brightening occurred at $\sim 16: 49-16: 50$ UT between N1 and P1, and a loop-like structure appeared at $\sim 16: 58$ UT in cool (1600, 171, and $304 \AA$ ) and hot (131 ̊) AIA channels, which suggests a wide range of plasma temperatures $(\sim 0.1-10 \mathrm{MK})$ within the loop. The bottom panels show a few dark threads (marked by an arrow) in the loop, which indicates cool chromospheric plasma within the loop.

To determine the plasma temperature $(\mathrm{T})$ and emission measure (EM) of the loop, we created peak temperature and EM maps using an automatic differential emission measure (DEM) code developed by Aschwanden et al. (2013). This code uses six channels of nearly simultaneous AIA images (171, 304, 193, 211,131 , and $94 \AA$ ) to derive the peak T and EM. We used sixchannel AIA images taken at $16: 58: 08$ UT. The peak T and EM map (Fig. 3) confirm that the bright loop contains a multitemperature plasma. We observed $\sim 10 \mathrm{MK}$ plasma near the two footpoints of the loop, while the middle section of the loop contained cool plasma ( $\leq 1 \mathrm{MK})$. AIA 304 and $171 \AA$ images did not show the hot portion $(\sim 10 \mathrm{MK})$ at the end of the loop, but the $131 \AA$ images does (see Figs. 2f,g,h). This indirectly suggests $\sim 10 \mathrm{MK}$ plasma at the opposite end of the heated loop (marked by a circle in Fig. 2h). The EM map also reveals high EM near the ends of the loop and low EM in the middle.

To investigate the particle acceleration or precipitation sites during the flare, we overlaid RHESSI X-ray 6-12 keV (red) and 12-25 keV (blue) contours (16:56:00-16:56:30 UT), and on top of an HMI magnetogram, AIA 1600, and $131 \AA$ images (Fig. 4). We reconstructed RHESSI X-ray images in the 6-12 keV and 12-25 keV using the CLEAN algorithm with $30 \mathrm{~s}$ integration time. The contour levels are at $85 \%$ and $95 \%$ of the peak intensity. Interestingly, the X-ray sources were co-spatial with sunspots $\mathrm{N} 1$ and $\mathrm{P} 1$, where the initial brightening in the AIA channels was detected. The AIA 131 and $1600 \AA$ images show the source locations at the footpoint (F1) of the heated loop. We do not see any X-ray source at the opposite footpoint (F2) of the loop. The energy release site is basically between N1 and $\mathrm{P} 1$.

The initial brightening and the location of the X-ray sources at footpoint F1 suggest that the magnetic reconnection most likely occurred at the same location between the oppositepolarity sunspots (N1 and P1). It is likely that a portion of the accelerated electrons produced during the reconnection (above F1) were injected into the loop and then precipitated at the remote footpoint F2, which caused chromospheric evaporation and heating of the footpoints of the loop up to $\sim 10 \mathrm{MK}$ temperatures.

\subsection{Oscillatory behavior in X-ray and EUV flux profiles}

The periodic variations of the X-ray flux in 6-12 keV energy range during the B6.0 flare indicate a periodic release of energy with five distinct cycles. To determine the source of the oscillation, we created a time-distance plot (Fig. 5a) using the AIA $304 \AA$ images (running-difference) along the slice S1 shown in Fig. $2 \mathrm{~g}$. The AIA $304 \AA$ channel images show the chromosphere and the transition region. The location of the slice was chosen to be immediately above the sunspots P1. The slice crosses footpoint F1 and the initial brightening site. The stack plot shows repeated brightenings occurring along S1 with five clear brightening features. These periodic brightenings are the result of repetitive magnetic reconnection between $\mathrm{N} 1$ and $\mathrm{P} 1$. These periodic disturbances can be interpreted by reconnectiongenerated outflows.

To check the upper photospheric $(T=5000 \mathrm{~K})$ and transition region ( $T=0.1 \mathrm{MK}$ ) responses of oscillatory energy release, we also selected a small region (Fig. 2e) that includes the sunspot $\mathrm{P} 1$ and N1 in AIA $1600 \AA$ intensity images and extracted the mean counts within it. Fig. 5b shows five AIA $1600 \AA$ peaks during the flare. We note that the AIA $1600 \AA$ plot shows the intensity oscillation (during the flare) over the sunspot region. These intensity oscillations are most likely the result of oscillatory reconnection at F1 during the B6.0 flare. 

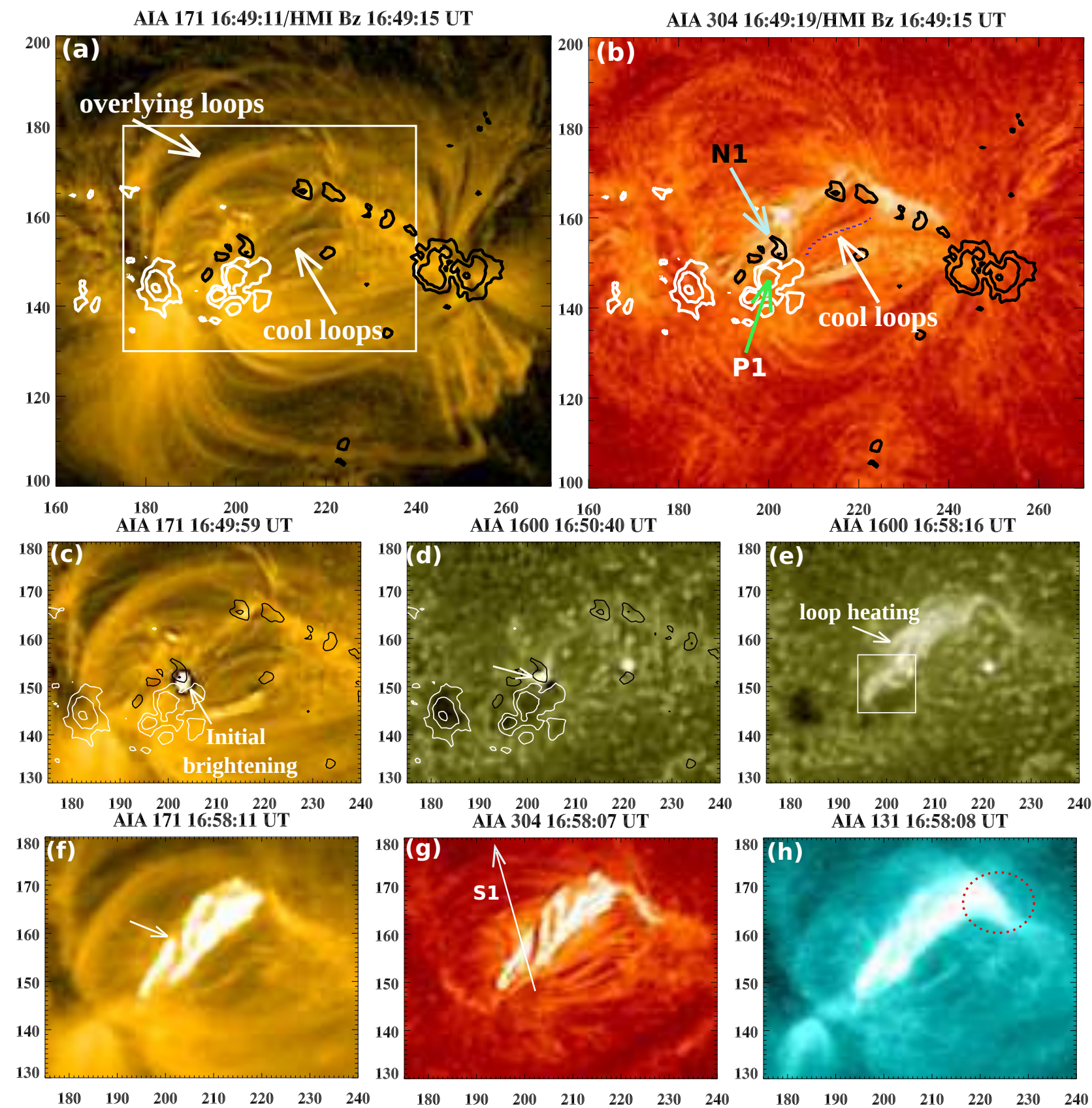

Fig. 2. a), b) AIA 171 and $304 \AA$ images before the onset of the first flare (B6.0). On these images are overlaid the HMI magnetogram contours of positive (white) and negative (black) polarities. The contour levels are $\pm 500, \pm 1000$, and \pm 1500 gauss. The rectangular (white) box indicates the size of the middle and bottom panels. P1 and N1 are positive and negative polarity sunspots. c), d) AIA 171 and $1600 \AA$ images showing the preflare brightening between N1 and P1. e)-h) The heated loop was observed in the cool (304, 171, and $1600 \AA$ ) and hot (AIA $131 \AA$ ) channels. $\mathrm{S} 1$ indicates the slice cut (over the footpoint of the loop) used to create the stack plot. The arrowhead shows the direction of the slice. The $x$ - and $y$-axes are labeled in arcsecs. The evolution of this flare is shown in an animation (i.e., AIA 171, 304, and $131 \AA$ A, bottom panels) available online.

As we described earlier, during the first X-ray peak of the B6.0 flare (16:51-16:54 UT), a B-class flare occurred in a different AR. The time-distance plot shows that the first burst in the 6-12 keV flux also receives a contribution from the studied B6.0 flare. Moreover, we can see a simultaneous $~ 3$-min oscillation in the AIA $1600 \AA$ and $304 \AA$ channels. The periodic brightenings above spots P1 and N1 strongly indicate the occurrence of repeated reconnections at the F1 footpoint of the heated loop. We also plot the RHESSI 6-12 keV flux (blue curve) along with the AIA 1600 mean counts, which shows a good correlation between $\mathrm{X}$-ray and EUV periodic energy release.

\subsection{Second C2.3 flare: merging of cool loops in the chromosphere and the flux rope formation}

To investigate the characteristics of the magnetic structure at the flare site, we used high-resolution NST TiO and VIS images taken before and during the $\mathrm{C} 2.3$ flare. The first B6.0 flare was not observed by the NST. The AIA resolution ( $0.6^{\prime \prime}$ per pixel) is not sufficient to resolve the smallest-scale chromospheric fine structures, therefore the NST observations $\left(0.029^{\prime \prime}\right.$ per pixel) are extremely useful for that purpose. In Fig. 6a we show a photospheric TiO image before the flare onset (17:16:05 UT) overlaid 

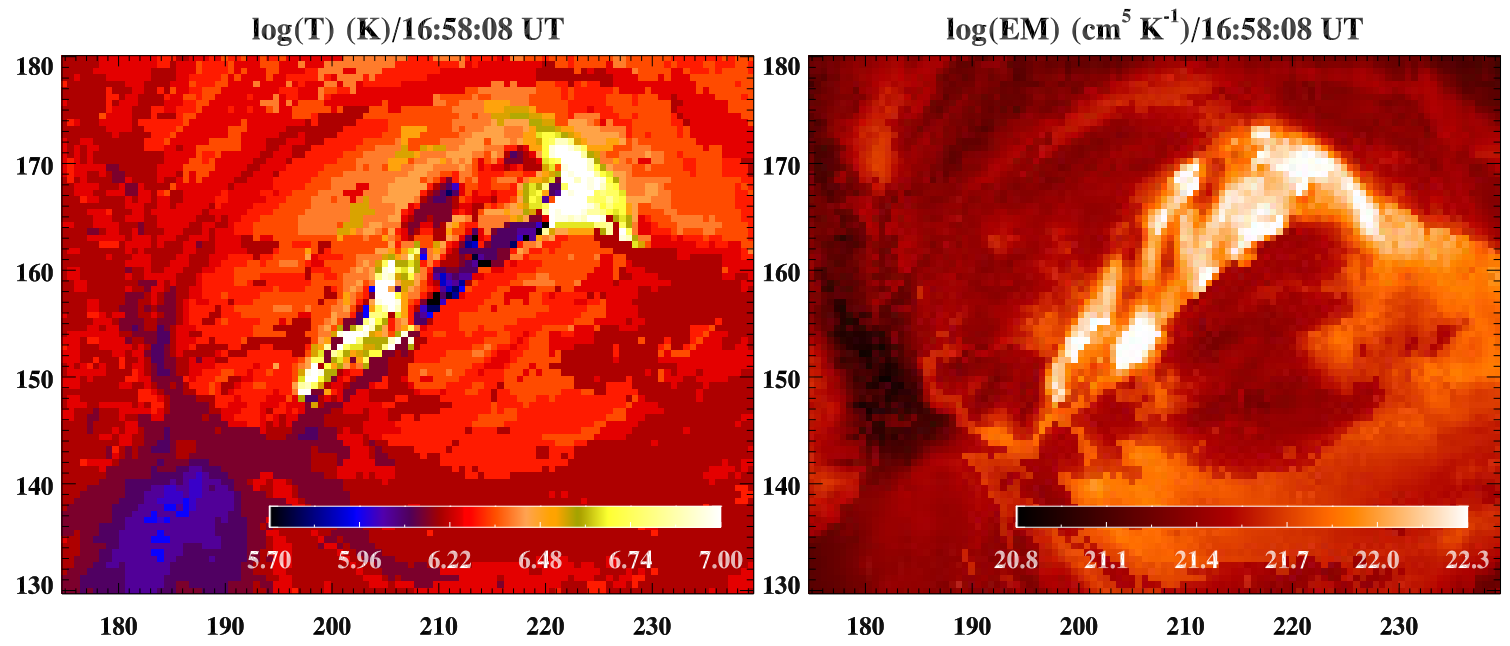

Fig. 3. Peak temperature and emission measure of the loop during the flare maximum (16:58:08 UT) derived from the near simultaneous AIA images in six channels. The loop contains multi-temperature plasma.
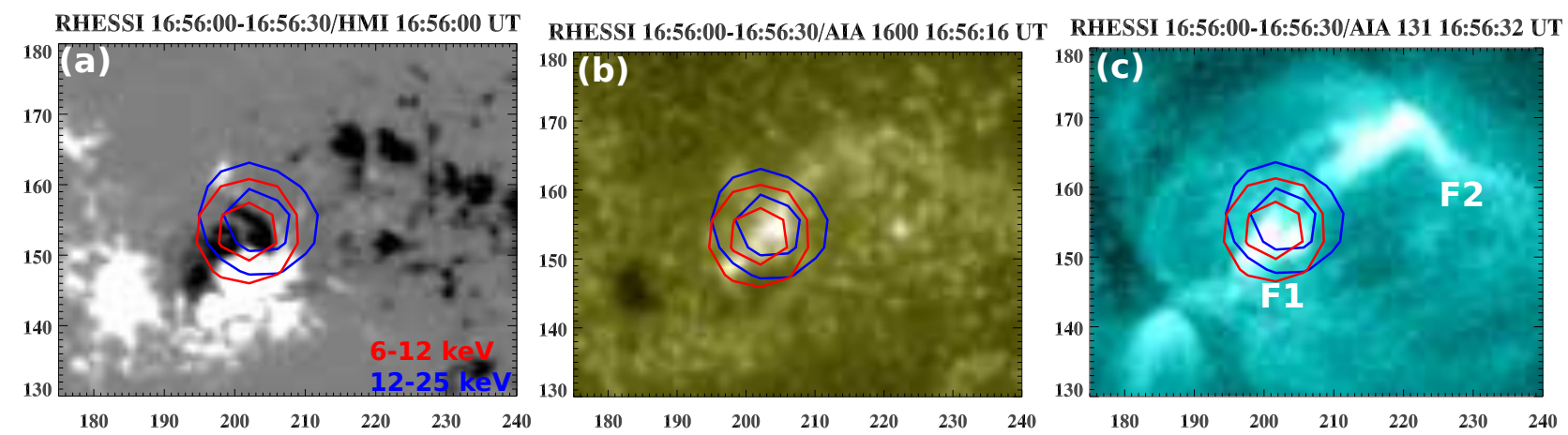

Fig. 4. RHESSI X-ray image contours (red: 6-12 keV, blue: $12-25 \mathrm{keV}$ ) overlaid on the HMI magnetogram, AIA 1600 , and $131 \AA$ images during the B6.0 flare. The contour levels are $85 \%$ and $95 \%$ of the peak intensity. F1 and F2 are the footpoints of the heated loop. The $x$ - and $y$-axes are labeled in arcsecs.

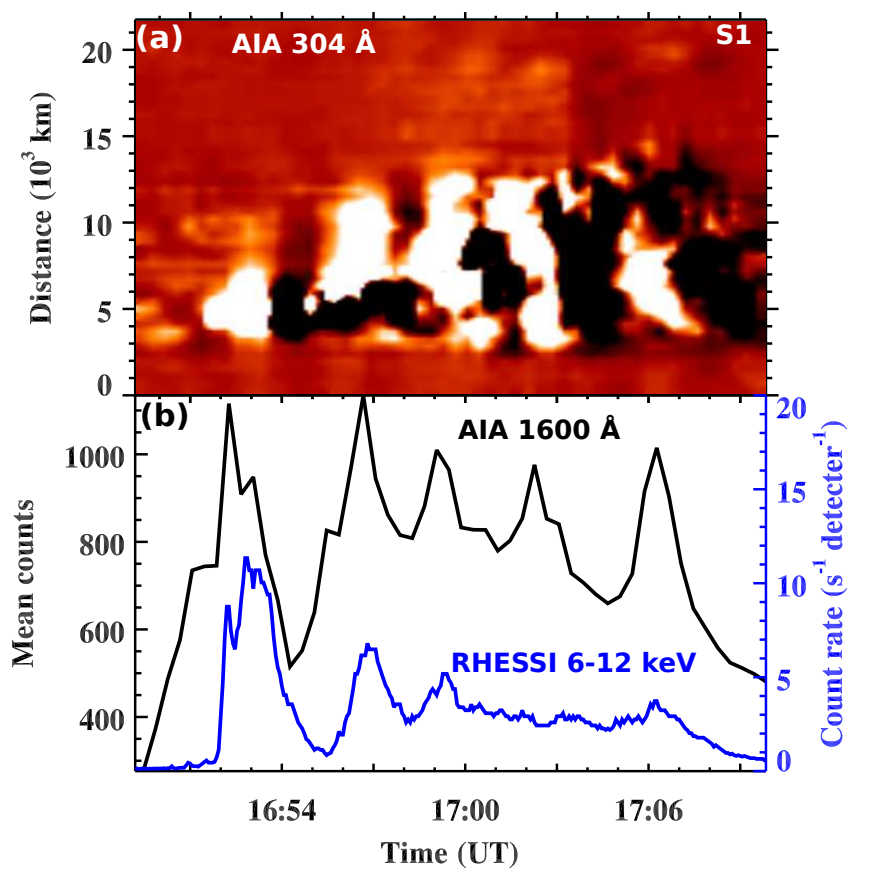

Fig. 5. a) Stack plot along slice $S 1$ (marked in Fig. 2g) using AIA $304 \AA$ running-difference images. b) AIA $1600 \AA$ mean counts extracted within a box region shown in Fig. 2e. The blue curve is the RHESSI X-ray flux in 6-12 keV channel. with HMI magnetic field contours of the positive (white) and negative (black) polarities. The positive- and negative-polarity sunspots are indicated by $\mathrm{P} 1, \mathrm{P} 2, \mathrm{P} 3, \mathrm{~N} 1, \mathrm{~N} 2$, and N3. The initial AIA flare brightening (see Fig. 2d) was observed between $\mathrm{N} 1$ and P1. The negative-polarity sunspot, N1, had an elongated shape. The $\mathrm{H} \alpha$ red wing image ( $\mathrm{H} \alpha+0.8 \AA, 6 \mathrm{~b})$ shows two loop systems (17:15:33 UT) at the flare site: loop L1 (red) connecting P3 and N1, and loop L2 (blue) spanning between P1 and N3. The two loops are highlighted with red and blue dotted lines, and their footpoint polarities are indicated by plus and minus signs. The cotemporal $\mathrm{H} \alpha$ line center image (6c) of the same region shows multiple chromospheric loops indicated by 1,2 , and 3 . We note that the loop 1 is the same as loop L1, whereas loops 2 and 3 connect different elements of positive flux (P2) in the photosphere. However, loop L2 has a single structure. After some time (17:23:08 UT, 6d), we observed the disappearance of the higher loop, loop 1, and merging of loops 2 and 3 into a single structure labeled L1. The eastern footpoint of the merged loop L1 in Fig. 6d is now connected to P2, which is different from L1, as shown in Fig. 6b. In this way, we finally have two loop systems in the chromosphere (L1 and L2) before the onset of the C2.3 flare.

Figure 7 displays a series of $\mathrm{H} \alpha$ line center, $\mathrm{H} \alpha-0.4 \AA$, and $\mathrm{H} \alpha-0.8 \AA$ flare images. During the initiation of the $\mathrm{C} 2.3$ flare, the initial brightening occurred at 17:29:35 UT at the footpoints of the penumbral filaments between $\mathrm{P} 1$ and $\mathrm{N} 1$ along with the 

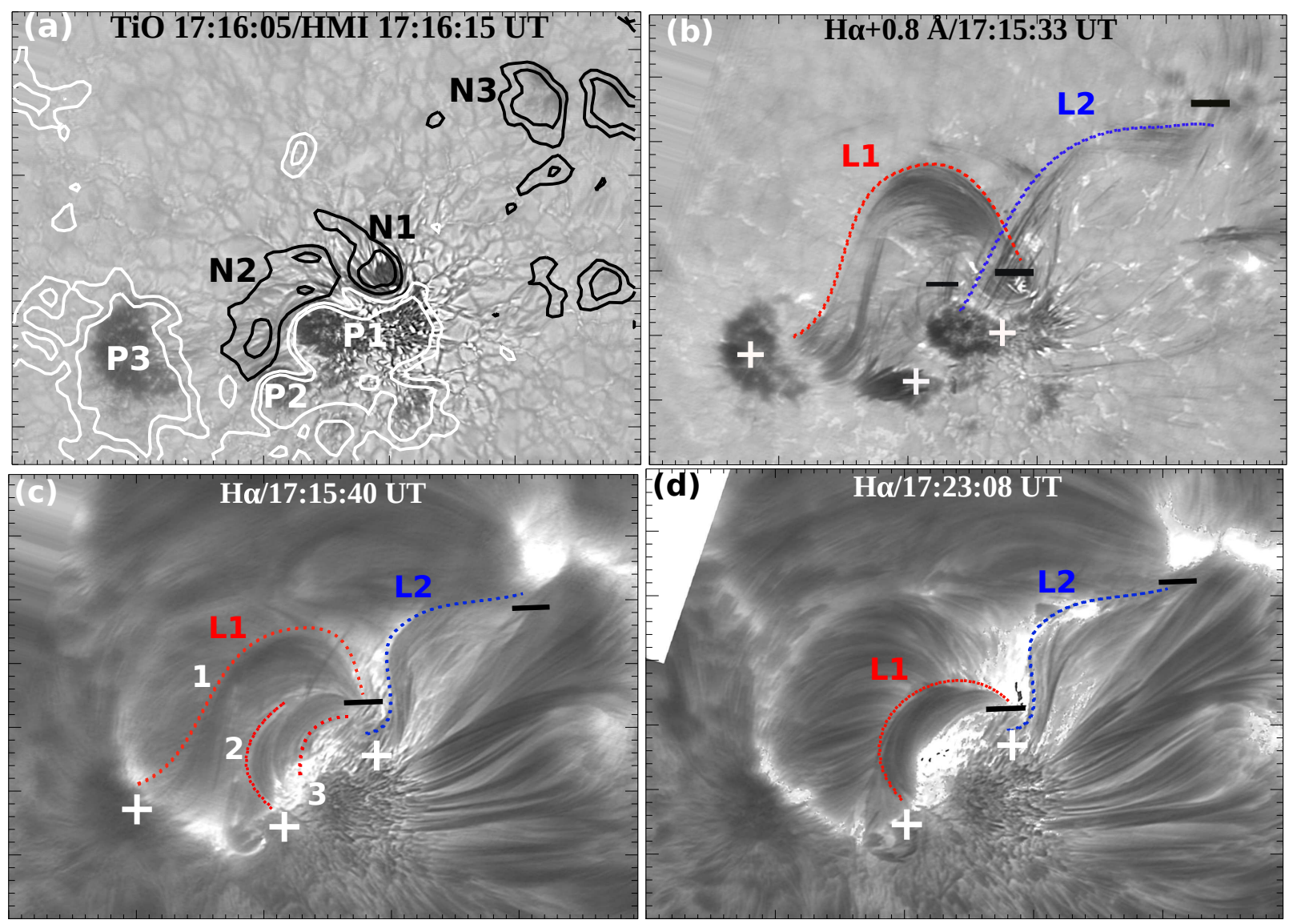

Fig. 6. a) NST TiO (7057 ̊) image overlaid by HMI magnetogram contours of positive (white) and negative (black) polarities. The contour levels are \pm 200 and \pm 500 gauss. b)-d) NST H $\alpha+0.8 \AA$ and $\mathrm{H} \alpha$ line center images showing two loop systems (L1 and L2) and their connectivity (marked by plus and minus signs). The size of each image a)-d) is $\sim 50^{\prime \prime} \times 35^{\prime \prime}$. Each division on the $x$-and $y$-axes is equal to $1^{\prime \prime}$.

activation of a cool loop L2 (blue dotted line). This is the same loop L2 as shown in Fig. 6. The loop was present before the C2.3 flare was triggered. The right footpoint of loop L2 was anchored in negative-polarity sunspot N3, while its left footpoint was anchored in a positive-polarity field between $\mathrm{N} 1$ and $\mathrm{P} 1$.

A clear interaction between the two loops L2 (blue) and L1 (red) can be observed starting at 17:32:11 UT, as shown by the chromospheric flare brightening that is seen between the interacting loops (Figs. 7b,c). In addition, we also note the counterclockwise rotation of the chromospheric loop structures above the positive-polarity sunspot P2 (marked by a yellow arrow in Fig. 7d). The viewing direction is toward the positive footpoint of the flux rope. In addition, we observe the counterclockwise rotation of P2 in the NST TiO (photospheric) movie. It is therefore likely that the rotation of $\mathrm{P} 2$ can increase the twist of the left-handed flux rope.

Loop L2 seems to display left-handed twisted threads, whereas loop L1 was smaller and seemed to have a potential field structure. After merging of loops L1 and L2, we see a combined twisted flux rope structure (at $\sim 17: 45$ UT). The interaction between L1 and L2 continued for about 15 min and produced a single merged flux rope with rotation in the counter-clockwise direction. The direction of the loop threads (marked by the yellow dotted line) suggests that the flux rope is also twisted in the lefthanded direction. The counterclockwise rotation corresponds to an increasing left-handed twist in the rope. The high-resolution NST images allowed us to observe details of a loop-loop interaction in the chromosphere for the first time.
In Fig. 8 we show the $\mathrm{C} 2.3$ flare brightening and the flux rope structure in the AIA channels. The AIA $171 \AA$ image (overlaid by HMI magnetogram contours) immediately before the flare onset (at 17:29:59 UT) shows the connectivity of the overlying loops at the flare site (Fig. 8a). Several overlying loops at different heights are evident. At least two sets of loops were clearly observed (i.e., low lying and higher loops). AIA 304 and $1600 \AA$ images at $\sim 17: 33$ UT clearly revealed the appearance of a hot loop with the onset of the impulsive brightening at footpoint F1 (Figs. 8b,c). The enlarged view of the interacting loops L1 and L2 has been discussed with the help of the NST H $\alpha$ images. The RHESSI X-ray image shows a single X-ray source (red: 6$12 \mathrm{keV}$, blue: $12-25 \mathrm{keV}$ ) located over the left footpoint of loop L2, exactly between the interacting loops L1 and L2 (Figs. 8b,c). The location of the sources suggests that the particle acceleration or precipitation site lies between the $\mathrm{N} 1$ and P1 sunspots (i.e., as a result of reconnection between L1 and L2). The RHESSI $\mathrm{X}$-ray movie does not show any shift of the X-ray source during the $\mathrm{C} 2.3$ flare energy release, suggesting a stationary particle precipitation site.

The AIA composite movie clearly shows the dynamics of the flux rope with its counterclockwise rotation. We see a resulting merged flux rope structure during 17:43-17:44 UT (Figs. 8d-f). The left-handed strands are observed in the flux rope structure (see AIA $304 \AA$ image). The flux rope could not escape from the overlying arcade loops (i.e., no removal of the overlying field), and the flux rope plasma drained back to the solar surface after the counterclockwise rotation. 

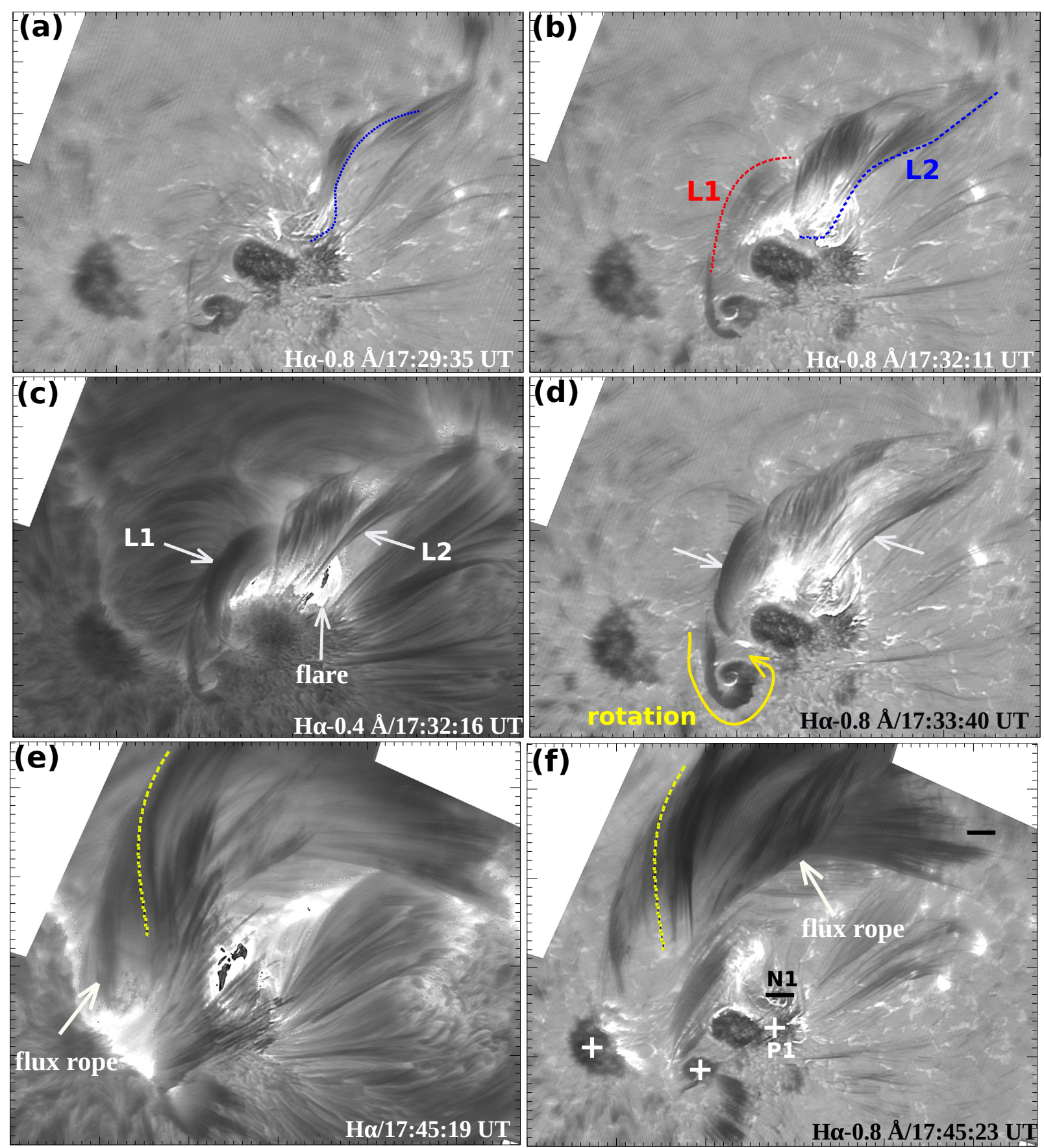

Fig. 7. a)-d) NST H $\alpha-0.8 \AA, \mathrm{H} \alpha-0.4 \AA$, and $\mathrm{H} \alpha$ line center images showing the interaction of two loop systems (L1 and L2) and the associated C-class flare. The size of each image a)-d) is $\sim 50^{\prime \prime} \times 35^{\prime \prime}$. e), f) NST H $\alpha$ line center and $\mathrm{H} \alpha-0.8 \AA$ images showing the flux rope produced by the interaction of cool loops. The size of each image e), f) is $\sim 57^{\prime \prime} \times 45^{\prime \prime}$. Each division on the $x$ - and $y$-axes is equal to $1^{\prime \prime}$. The coalescence of loops (L1 and L2) and formation of a resulting flux rope is shown in the $\mathrm{H} \alpha$ line center (panel e)) and $\mathrm{H} \alpha-0.8 \AA$ (panels a), b), d), and f)) animations available online.

To study the dynamics or kinematics of the flux rope along with the signatures of magnetic reconnection, we analyze AIA $304,171,131$, and $1600 \AA$ images during the $\mathrm{C} 2.3$ flare (17:3017:45 UT). Figure 9 displays the stack plots created along the slices S2, S3, S4, and S5 marked in the AIA 304 and $131 \AA$ images. The slices S2 and S4 are across the flux rope and show the two rise phases, (i) a slow rise with $\sim 20 \mathrm{~km} \mathrm{~s}^{-1}$ during 17:30-17:36 UT and (ii) a fast-rise with $52 \mathrm{~km} \mathrm{~s}^{-1}$ during 17:3617:41 UT. The flux rope showed rotation in its positive footpoint area and could not proceed after reaching a projected height of $\sim 30 \mathrm{Mm}$. We included Fermi GBM 6-12 keV X-ray flux in order to compare the flux rope dynamics along with the particle acceleration (X-ray bursts). We see the first energy-release or burst (17:30-17:35 UT) during the interaction of L1 and L2 in the chromosphere as seen in the NST $\mathrm{H} \alpha$ images. During the interaction time, the resulting flux rope rises slowly with $\sim 20 \mathrm{~km} \mathrm{~s}^{-1}$. Later, we see multiple X-ray bursts with a fast rise of the rope and strong chromospheric brightenings below the flux rope during 17:36-17:41 UT. Interestingly, we see multiple loops and brightening patches (in the AIA $131 \AA$ A channel, marked within ellipse) moving above the reconnection site (i.e., below the flux rope), suggesting the occurrence of oscillatory 

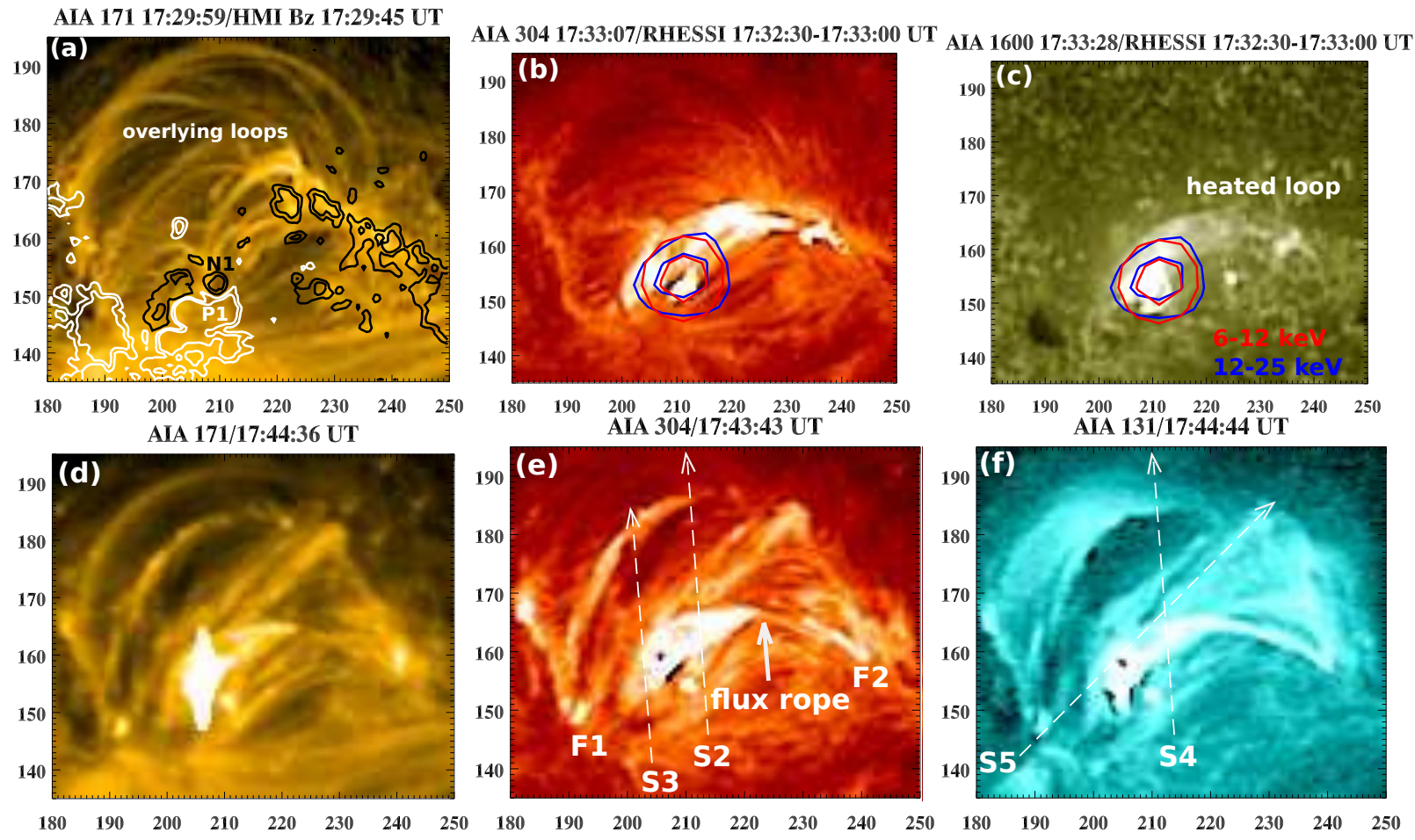

Fig. 8. a) AIA $171 \AA$ image with HMI magnetogram contours of positive (white) and negative (black) polarities. The contour levels are \pm 200 and \pm 500 gauss. b)-c) RHESSI X-ray contours (red: 6-12 keV, blue: $12-25 \mathrm{keV}$ ) overlaid on the AIA 304 and $1600 \AA$ images. The contour levels are $85 \%$ and $95 \%$ of the peak intensity. d) -f) Observation of the flux rope in different AIA channels (304, 171, and 131 $\AA$ ). S2, S3, S4, and S5 are the slices used to create the stack plots. F1 and F2 are the new footpoints of the flux rope. The oscillatory reconnection and associated flux rope formation with counterclockwise rotation is shown by a composite movie (AIA 171, 304, and $131 \AA$, bottom panels) available online.

reconnection-generated outflows. The fast rise of the flux rope correlates with the quasi-periodic variation in the X-ray flux (17:36-17:41 UT), which suggests quasi-periodic reconnection among the loops during the expansion of the flux rope. We also see counterclockwise motion $\left(\sim 72 \mathrm{~km} \mathrm{~s}^{-1}\right)$ of the chromospheric structure (or loops) rooted in $\mathrm{P} 2$ that merged with the new footpoint of the flux rope.

The comparison of the RHESSI X-ray sources location with the periodic brightening site (within ellipse) observed in the AIA $131 \AA$ channel (Fig. 9d) suggests the occurrence of oscillatory reconnection (above the sunspots $\mathrm{P} 1$ and $\mathrm{N} 1$ ) during the interaction of the cool loops L1 and L2. The AIA 304, 171, and $131 \AA$ composite movie shows the initial brightening between L1 and L2, followed by an expansion of the rope and associated brightening to the east. In addition, the outflows observed in the AIA $131 \AA$ channel are quasi-periodic as well and the oscillation period $(\sim 0.5-1 \mathrm{~min})$ is different from the preceding B6.0 flare.

\subsection{Magnetic field evolution}

Figure 10a displays the complete view of AR NOAA 12353, and the first brightening starts within the box region (red) between $\mathrm{N} 1$ and P1. To check the flux emergence or cancellation during the flare, we analyzed HMI magnetograms for the interval of 16:30 UT to 18:30 UT. The HMI movie shows the shearing motions and rotation of the negative-polarity sunspot (N1) that continuously moved southward and pushed the positive-polarity region (P1). Interestingly, the HMI movie also shows rapid cancellation of both negative and positive flux when the loop coalesces.

In order to quantify the flux cancellation, we extracted positive, negative, and total magnetic fluxes within a rectangular box region that encloses the flare site. Figure $10 \mathrm{~b}$ shows the positive (red), absolute negative (blue), and total (black) magnetic flux profiles. The GOES soft X-ray flux profile in the 1-8 $\AA$ channel is also included (bottom panel) to compare the timing of the flares. The rapid flux cancellation of both polarities started during the B6.0 flare and continued until the end of the $\mathrm{C} 2.3$ flare. The rapid cancellation of negative or total flux proceeded even when there were no flares in progress ( 17:08-17:28 UT), when loops (labeled 1, 2, and 3) merged in the NST $\mathrm{H} \alpha$ images (Fig. 6). The positive flux cancellation was not as large as the negative flux cancellation. The negative flux changed from $\sim 3.7 \times 10^{20} \mathrm{Mx}$ to $\sim 2.7 \times 10^{20} \mathrm{Mx}$ ( $\sim 27 \%$ decrease). The positive flux decreased only by $5 \%$ from $\sim 1.25 \times 10^{21} \mathrm{Mx}$ to $\sim 1.20 \times 10^{21} \mathrm{Mx}$. The total unsigned flux changed from $\sim 1.62 \times 10^{21} \mathrm{Mx}$ to $\sim 1.47 \times 10^{21} \mathrm{Mx}$. After the flare, the magnetic flux changes were nearly negligible. To estimate the flux cancellation rate, we performed a linear fit to the time profiles of magnetic fluxes between 17:18 UT and 17:42 UT and calculated the slopes. The estimated total unsigned, positive, and negative flux cancellation rates are comparable and equal to $3.44 \times 10^{20} \mathrm{Mx} \mathrm{h}^{-1}, 1.57 \times 10^{20} \mathrm{Mx} \mathrm{h}^{-1}$, and $1.86 \times 10^{20} \mathrm{Mx} \mathrm{h}^{-1}$, respectively.

The evolution of the magnetic field before, during, and after the flare is shown in selected magnetograms (Figs. 10c-f). The detachment of the tail of the tadpole-like negative sunspot N1 (blue rectangle, marked by an arrow) is a result of flux cancellation during the flare. The motion of the negative-polarity sunspot $\mathrm{N} 1$ and its cancellation with the ambient positive-polarity region P1 (red arrow) is clearly observed.

To investigate the details of the morphological changes in the photospheric magnetic structures during the flares, we used NST TiO (7055 $\AA$ ) images. Figure 11 displays the cotemporal HMI magnetogram contour over the TiO images at the start time 
(17:30:05 UT) and after the flare (17:50:07 UT). The cancellation of N1 and P1 is clearly visible. Initially, N1 had a tail structure, and we see the tail disappears during the flare. Interestingly, we also observe the shrinkage of N1 (in the TiO movie) during the flare. The magnetogram contours of $\mathrm{N} 1$ over $\mathrm{TiO}$ images also show the reduced umbral area (i.e., collapse) of the spot N1. Furthermore, we see cancellation of an elongated structure in P1, which is marked by the red arrow.

Figure 12a displays the potential field extrapolation of the AR using the HMI magnetogram (at 16:48:38 UT) before the flare trigger. The closed field lines are shown in blue and the open field lines in white. The bunches of overlying field lines above the flare site are clearly visible. The connectivity of the field lines between P1 and N3, and between P2 and N1 is very similar to the loops observed in the $\mathrm{H} \alpha$ and EUV images.

To check the variation in the horizontal component of the overlying magnetic field with respect to height, we estimated the decay index $n=-\mathrm{d}\left(\log B_{\mathrm{h}}\right) / \mathrm{d}(\log H)$, where $B_{\mathrm{h}}$ is the horizontal magnetic field strength and $H$ is the height above the solar surface. We calculated the mean value of $B_{\mathrm{h}}$ above the PIL between $\mathrm{N} 1$ and P1 (marked in Fig. 10a). Figure 12b displays the decay index vs. height above the PIL. According to the torus instability criteria (Kliem \& Török 2006; Olmedo \& Zhang 2010), the threshold value of the decay index should be $\geq 1.5$. The threshold value of the decay index (1.5) can be achieved at a height of $\sim 120 \mathrm{Mm}$. The flux rope therefore should attain this height for a successful eruption. In addition, the AIA $304 \AA$ stack plot (Fig. 9) reveals that the flux rope attains a height (projected) of about $25-30 \mathrm{Mm}$. The flux rope is most likely stopped by the strong overlying field of the AR and was unable to satisfy the condition for the torus instability, which resulted in the failed eruption.

\section{Summary and discussion}

We presented high-resolution multiwavelength observations of two homologous flares that were accompanied by an oscillatory energy release associated with formation and disruption of a flux rope. The main results of this study are summarized below.

We observed interaction and reconnection of cool chromospheric $\mathrm{H} \alpha$ loops. The loop interaction (merging of the loops) was associated with the formation of a left-handed flux rope along with a rapid photospheric flux cancellation observed by the HMI. The flux rope showed rotation in the counterclockwise direction, and it failed to erupt owing to the presence of strong overlying fields. We present the following evidence of reconnection at footoint F1: (i) clear interaction between loops L1 and L2, as shown in the high-resolution NST images. L2 starts to activate or appear during the reconnection between L1 and L2. We noted a $\mathrm{H} \alpha$ brightening between L1 and L2. (ii) The location of the X-ray sources suggests that the particle acceleration or precipitation site lies above and between N1 and P1. (iii) A rapid photospheric flux cancellation between $\mathrm{P} 1$ and $\mathrm{N} 1$ during the interaction is strong evidence of reconnection between the chromospheric loops.

The rotation in the counterclockwise direction indicates a left-handed flux rope. The strands in the flux rope confirm the left-handed twist. The clockwise rotation of N1 increases the right-handed twist, whereas the counterclockwise rotation of P2 adds left-handed twist in the coronal flux that is rooted in these spots. The $\mathrm{H} \alpha$ images indicate left-handed flux in the formed flux rope, which suggests a more dominant role of the rotation of P2. Moreover, P2 contains more magnetic flux than N1.

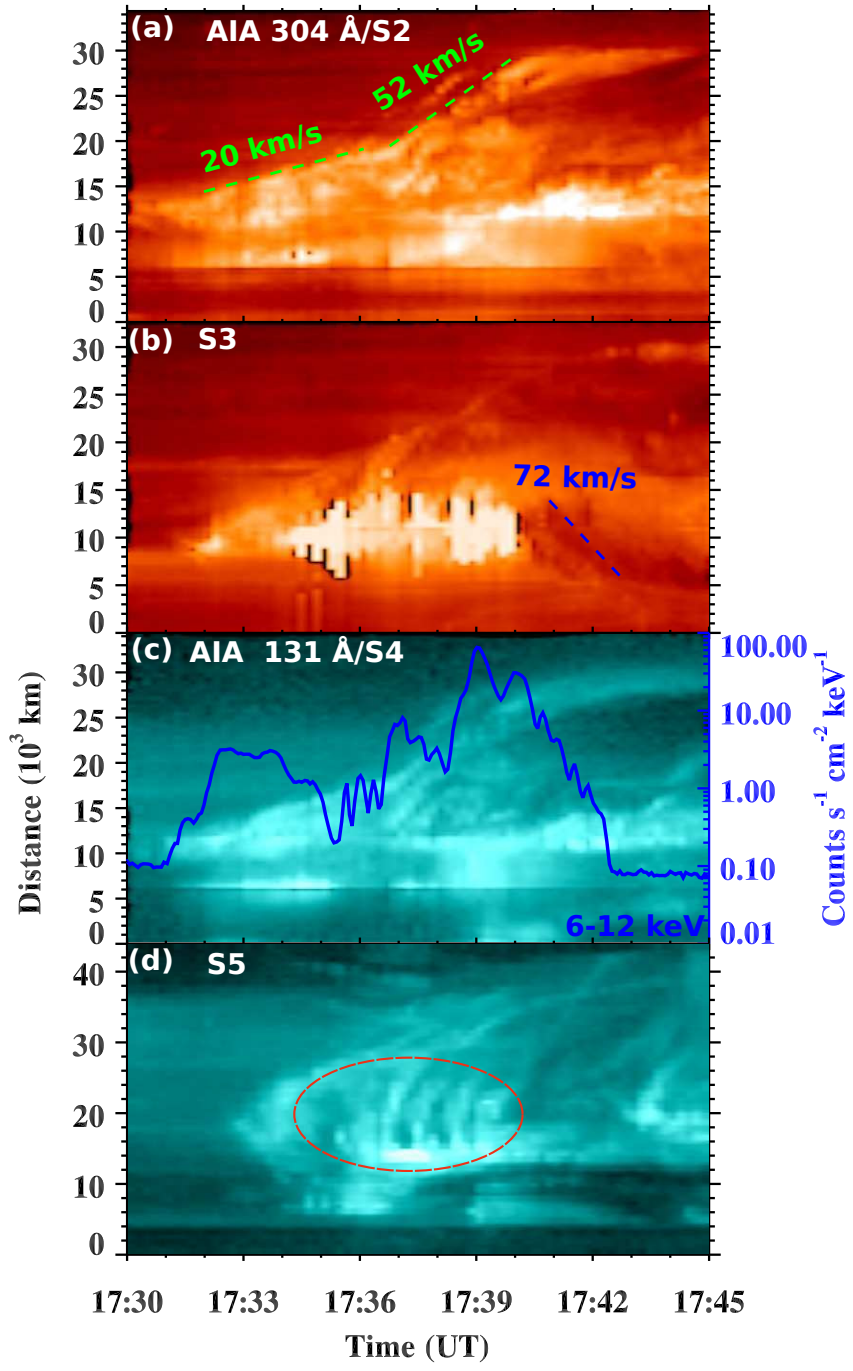

Fig. 9. Stack plots along the slices S2, S3, S4, and S5 using AIA 304 and $131 \AA$ images. The Fermi GBM X-ray flux in the $6-12 \mathrm{keV}$ channel is also included in panel c). The periodic rise of multiple reconnected loops is shown within a dashed ellipse (panel d)). The cotemporal X-ray bursts (panel c)) and strong chromospheric brightening in the AIA $304 \AA$ channel (panel b)) indicate the particle acceleration and precipitation during the periodic rise of reconnecting loops.

Yurchyshyn et al. (2001) reported one-hour-long flux cancellation in the sunspot moat that proceeded at a rate of $3 \times$ $10^{19} \mathrm{Mx} \mathrm{h}^{-1}$. They interpreted the cancellation to be due to photospheric reconnection between small flux elements and noted its possible connection to flares. Here we observed flux cancellation between two small sunspots not only during the flare, but also 15-20 min before the C2.3 flare. In our case, the total absolute flux cancellation rate is $\sim 3.44 \times 10^{20} \mathrm{Mx} \mathrm{h}^{-1}$, which is almost 10 times longer than that reported by Yurchyshyn et al. (2001). Green et al. (2011) reported cancellation of the total unsigned flux of $\sim 1.17 \times 10^{21} \mathrm{Mx}$ ) at PIL in a decaying AR during $\sim 2.5$ days preceding a CME and suggested that the major flux cancellation was responsible for the formation of an S-shaped sigmoid or flux rope (before the eruption). However, here we observed rapid flux cancellation (total unsigned flux $\sim 1.7 \times 10^{20} \mathrm{Mx}$ ) within only $\sim 20 \mathrm{~min}$, which is much faster (but smaller) than that observed by Green et al. (2011). Therefore, we relate the rapid and major flux cancellation to the formation of the flux rope. This much more rapid flux cancellation has hardly 


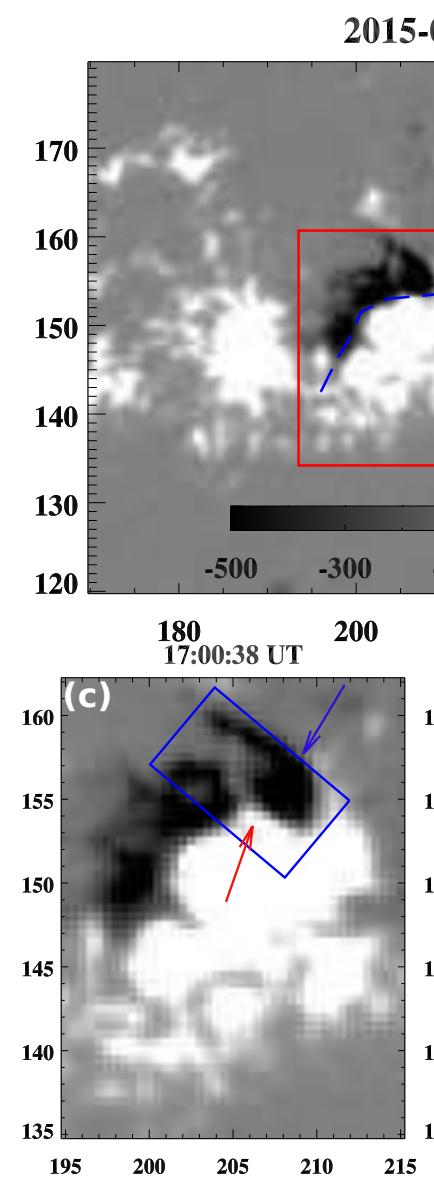

2015-05-23/16:30:38 UT
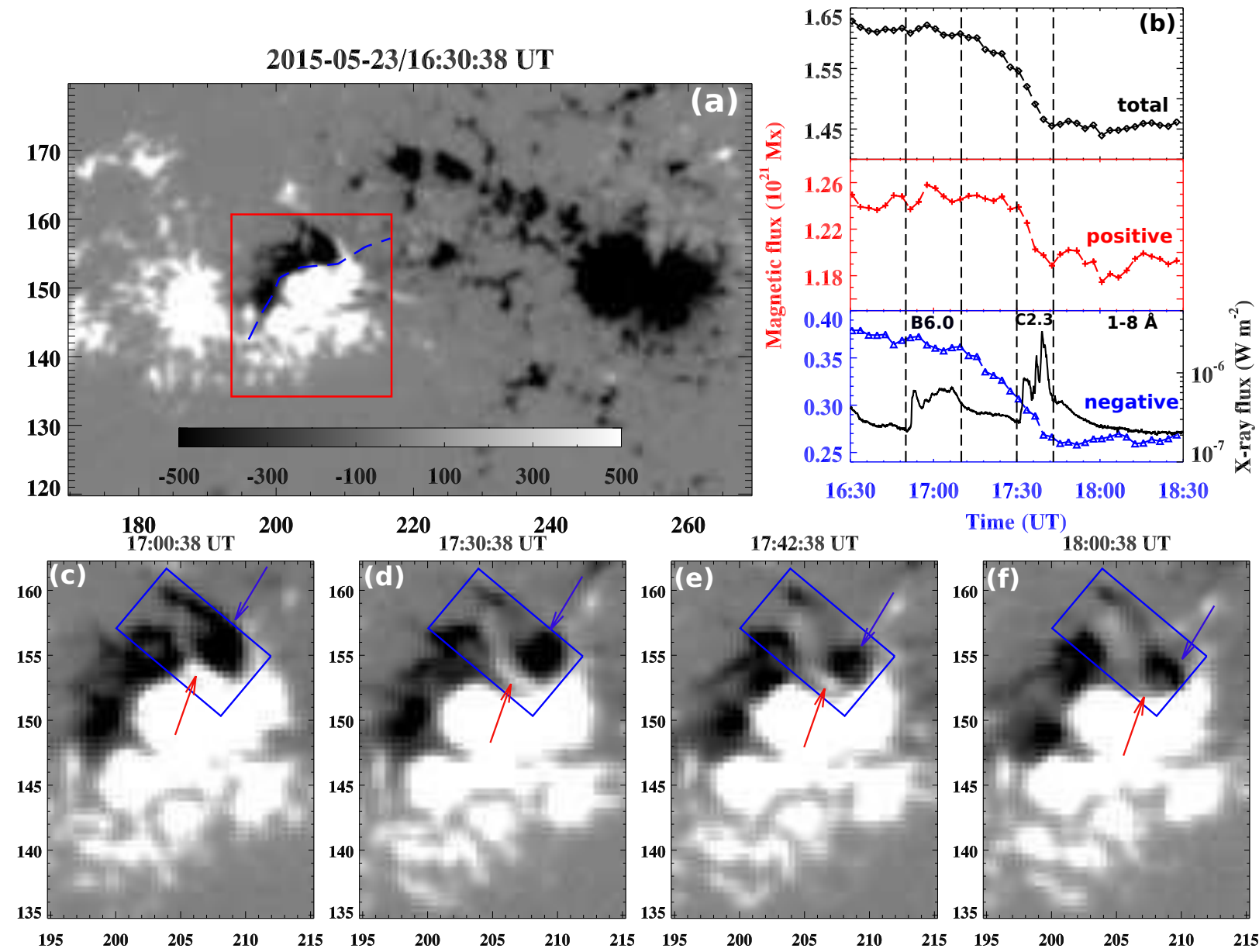

Fig. 10. a) HMI magnetogram of AR NOAA 12353. b) Positive (red), absolute negative (blue), and total (black) flux profiles extracted from the rectangular box (red) region shown in the left panel. The GOES soft X-ray flux profile in 1-8 $\AA$ channel is also plotted with negative flux profile. The vertical dashed lines indicate the B6.0 and C2.3 flare durations. c)-f) Selected HMI magnetograms of the flare site showing flux cancellation (within blue rectangular box). The $x$ - and $y$-axes are labeled in arcsecs. The shear motion and rapid flux cancellation between N1 and P1 is shown in the HMI magnetograms movie (bottom panels) available online.

ever been observed before. In addition, the magnetic flux in interplanetary magnetic clouds lies between $10^{20} \mathrm{Mx}$ to $10^{22} \mathrm{Mx}$ (Qiu et al. 2007). Here we observed a small-scale flux rope (not a large-scale flux rope). The magnetic flux associated with the rope may therefore be on the order of $10^{20} \mathrm{Mx}$ (lower limit).

Without the use of high-resolution NST images, AIA observations could have been interpreted as activation of a preexisting flux rope that is heated during the first B6.0 flare and tries to erupt during the second C2.3 flare. However, we do not see any preexisting filament or flux rope before the onset of the first B6.0 flare. We only observe a system of cool chromospheric loops (connecting P1 and N3) in the AIA 304, $171 \AA$, and labeled L2 in the NST H $\alpha$ images. We are able to see clear interaction of cool loops at the PIL and the development of a twisted flux rope associated with a rapid flux cancellation, and therefore we suggest that the flux rope was most likely developed or formed through magnetic reconnection of the loops associated with $\mathrm{N} 1$ and $\mathrm{P} 1$ sunspots. This result supports the previous report of the formation of an S-shaped flux rope through chromospheric reconnection (Kumar et al. 2015). However, we did not observe fast flux cancellation like this in our previous studies. In the tether-cutting reconnection model (Moore et al. 2001), the reconnection between the sheared arcade loops (in the corona) results in the formation of a coronal flux rope. Here we report the reconnection between the cool $\mathrm{H} \alpha$ loops in the chromosphere that forms a flux rope.

The dynamic radio spectrum $(25-180 \mathrm{MHz})$ observed at Sagamore Hill ${ }^{1}$ radio station does not show any radio signatures during either of the two flares. No type III radio burst was observed during the two flares, which suggests the absence of particle acceleration into the interplanetary medium along the open field lines. Most of the accelerated particles were confined along the closed loops within the AR. A part of accelerated electrons most likely followed the closed field lines that led to their precipitation at the opposite footpoint of the reconnecting twisted loop. This resulted in chromospheric evaporation and heating of the loops to a temperature of $\sim 10 \mathrm{MK}$.

The height (projected) of the overlying arcades in the $171 \AA$ channel exceeds the flux rope height $(\sim 25-30 \mathrm{Mm})$ during the C2.3 flare. In addition, the threshold value of the decay index above the PIL (1.5) could be achieved at a height of $\sim 120 \mathrm{Mm}$. Therefore, the flux rope did not attain the height of torus instability. Kumar \& Cho (2014) reported that a kinked small filament reached a height of $\sim 60 \mathrm{Mm}$ and was unable to erupt because of the interaction with overlying arcade loops. In addition, when the flux rope is very small, the total energy of

\footnotetext{
1 http://www.ngdc. noaa.gov/stp/space-weather/ solar-data/solar-features/solar-radio/
} 
TiO 17:16:05/HMI 17:16:15 UT

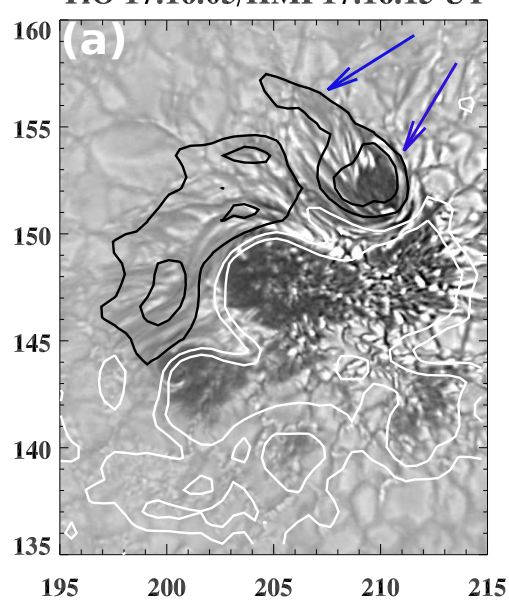

TiO 17:30:05/HMI 17:30:30 UT

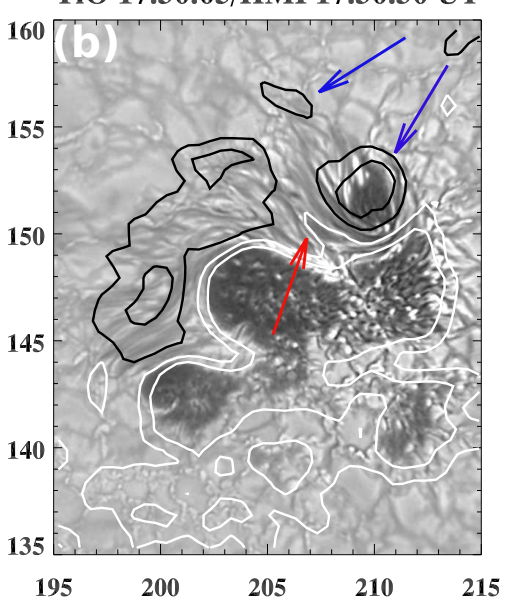

TiO 17:50:07/HMI 17:50:00 UT

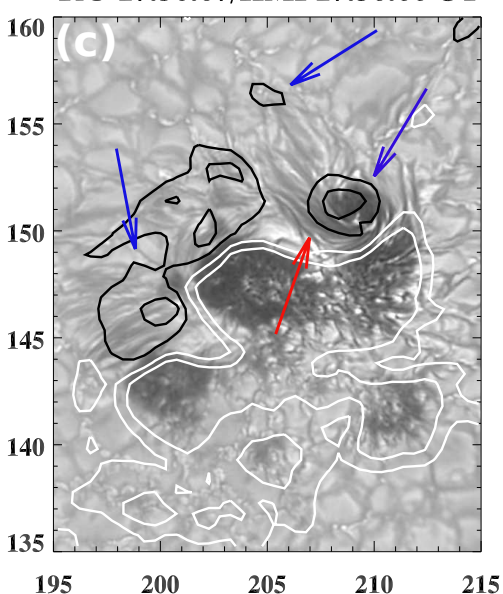

Fig. 11. NST TiO (7057 Å) images overlaid by HMI magnetogram contours of positive (white) and negative (black) polarities. The contour levels are \pm 200 and \pm 500 gauss. Flux cancellation and shrinkage of negative-polarity sunspots are marked by arrows. The $x$ - and $y$-axes are labeled in arcsecs.
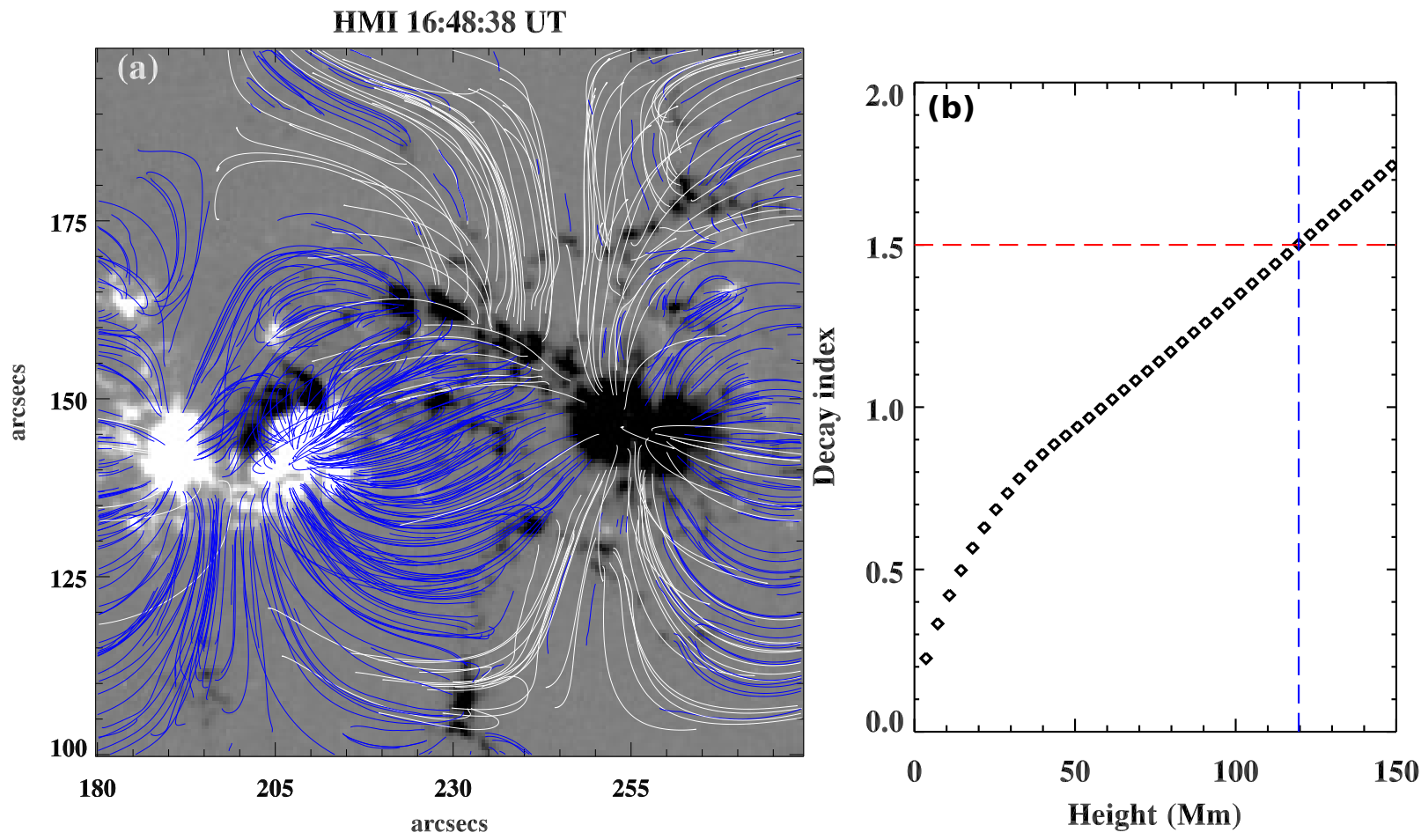

Fig. 12. a) Potential field extrapolation of the AR using HMI magnetogram at 16:48:38 UT. The closed and open field lines are shown in blue and white, respectively. b) Variation of the decay index with respect to height (Mm) above the PIL. The red horizontal dashed line indicates the threshold value of the decay index (1.5) at a height of $\sim 120 \mathrm{Mm}$ (blue vertical dashed line).

the rope may not be sufficient to overcome or open the overlying field and could show a counterclockwise rotation within the overlying arcade loops. After the twist release, the plasma falls back to the solar surface.

The RHESSI X-ray sources (6-12 keV and 12-25 keV) do not show any significant motion during the flare, that is, particle acceleration or energy release sites remain stationary (between $\mathrm{P} 1$ and N1) during the flare. In addition, the RHESSI X-ray source (6-12 keV) was located above the sunspots P1 and N1, which is consistent with the intensity oscillation site observed in the EUV channels (1600 and $304 \AA$ ). During the second C2.3 flare, we observed the cancellation of the elongated penumbral field (see the TiO images) between $\mathrm{N} 1$ and P1. The footpoint of the loop L1 was rooted in the penumbral field of positive-polarity
P1. Both flares reveal an oscillatory behavior in the X-ray and EUV flux profiles that most likely indicates the occurrence of repeated reconnection by the loop coalescence between sunspots P1 and N1.

Previous observations of reconnection between loops in the chromosphere showed the standard flare reconnection in a current sheet below erupting flux (Yang et al. 2015; Xue et al. 2016), while here the process of tether-cutting and flux rope formation was observed for the first time in the chromosphere.

In conclusion, we have reported the direct observations of reconnection between chromospheric loops associated with rapid flux cancellation and flux rope formation with counterclockwise rotation. The hot flux ropes (observed in the AIA 131 and $94 \AA$ ) are generally formed by reconnection among the coronal 
loops (e.g, Kumar \& Cho 2014). Here, we reported the formation of a cool flux rope (H $\alpha$, AIA 304 and $171 \AA$ ) by multiple reconnection between the chromospheric loops. These observations support the model of flux rope formation proposed by van Ballegooijen \& Martens (1989). Such a clear loop coalescence associated with flux rope formation in a short interval ( $\sim 30 \mathrm{~min}$ ) has not been reported before. Future multiwavelength studies with high-resolution observations (NST along with IRIS and SDO) will shed more light on the issues related to the flux rope formation and eruption.

Acknowledgements. We thank the referee for constructive comments and suggestions that improved the manuscript considerably. SDO is a mission fo NASA's Living With a Star (LWS) program. The SDO data were (partly) provided by the Korean Data Center (KDC) for SDO in cooperation with NASA and SDO/HMI team. RHESSI is a NASA Small Explorer. BBSO operation is supported by NJIT, US NSF AGS-1250818 and NASA NNX13AG14G and NST operation is partly supported by the Korea Astronomy and Space Science Institute and Seoul National University, and by strategic priority research program of CAS with Grant No. XDB09000000. H.W. is supported by US NSF under grants AGS 1348513 and 1408703, and NASA under grants NNX13AG13G/NNX16AF72G . K.-S. Cho acknowledges support by a grant from the US Air Force Research Laboratory, under agreement number FA 238614-1-4078 and by the "Planetary system research for space exploration" from KASI. This work was supported by the "Operation of Korea Space Weather Center" of KASI and the KASI basic research funds. This work was conducted as part of the effort of NASA's Living with a Star Focused Science Team "Jets" NASA LWS NNX11AO73G grant. V.Yu. acknowledges support from NSF AGS1146896, AFOSR-FA9550-15-1-0322 grants and Korea Astronomy and Space Science Institute. P.K. thanks Prof. Valery M. Nakariakov for several helpful discussions.

\section{References}

Antiochos, S. K. 1998, ApJ, 502, L181

Aschwanden, M. J., Boerner, P., Schrijver, C. J., \& Malanushenko, A. 2013, Sol. Phys., 283, 5

Aulanier, G., Török, T., Démoulin, P., \& DeLuca, E. E. 2010, ApJ, 708, 314

Bạk-Stęślicka, U., Gibson, S. E., Fan, Y., et al. 2013, ApJ, 770, L28

Bothmer, V., \& Schwenn, R. 1998, Annales Geophysicae, 16, 1

Burlaga, L. F., Klein, L., Sheeley, Jr., N. R., et al. 1982, Geophys. Res. Lett., 9, 1317

Canfield, R. C., Hudson, H. S., \& McKenzie, D. E. 1999, Geophys. Res. Lett., 26, 627

Chen, P. F. 2011, Liv. Rev. Sol. Phys., 8, 1

Chen, P. F., \& Shibata, K. 2000, ApJ, 545, 524

Cheng, X., Zhang, J., Liu, Y., \& Ding, M. D. 2011, ApJ, 732, L25

Einaudi, G., \& van Hoven, G. 1983, Sol. Phys., 88, 163

Fan, Y., \& Gibson, S. E. 2004, ApJ, 609, 1123

Gary, G. A., \& Moore, R. L. 2004, ApJ, 611, 545

Gibson, S. E., Fan, Y., Török, T., \& Kliem, B. 2006, Space Sci. Rev., 124, 131
Gopalswamy, N., Yashiro, S., Michalek, G., et al. 2005, Geophys. Res. Lett., 32, 12

Gosling, J. T., Birn, J., \& Hesse, M. 1995, Geophys. Res. Lett., 22, 869

Green, L. M., \& Kliem, B. 2009, ApJ, 700, L83

Green, L. M., Kliem, B., \& Wallace, A. J. 2011, A\&A, 526, A2

Hood, A. W., \& Priest, E. R. 1981, Geophys. Astrophys. Fluid Dyn., 17, 297

Karpen, J. T., Antiochos, S. K., \& DeVore, C. R. 2012, ApJ, 760, 81

Kliem, B., \& Török, T. 2006, Phys. Rev. Lett., 96, 255002

Kliem, B., Titov, V. S., \& Török, T. 2004, A\&A, 413, L23

Kumar, P., \& Cho, K.-S. 2014, A\&A, 572, A83

Kumar, P., \& Innes, D. E. 2013, Sol. Phys., 288, 255

Kumar, P., Srivastava, A. K., Filippov, B., \& Uddin, W. 2010, Sol. Phys., 266, 39

Kumar, P., Manoharan, P. K., \& Uddin, W. 2011a, Sol. Phys., 271, 149

Kumar, P., Srivastava, A. K., Filippov, B., Erdélyi, R., \& Uddin, W. 2011b, Sol. Phys., 272, 301

Kumar, P., Cho, K.-S., Bong, S.-C., Park, S.-H., \& Kim, Y. H. 2012, ApJ, 746, 67

Kumar, P., Yurchyshyn, V., Wang, H., \& Cho, K.-S. 2015, ApJ, 809, 83 Lemen, J. R., Title, A. M., Akin, D. J., et al. 2012, Sol. Phys., 275, 17 Lin, R. P., Dennis, B. R., Hurford, G. J., et al. 2002, Sol. Phys., 210, 3 Liu, R., Liu, C., Wang, S., Deng, N., \& Wang, H. 2010, ApJ, 725, L84 Longcope, D. W., \& Beveridge, C. 2007, ApJ, 669, 621

Manoharan, P. K. 2010, Sol. Phys., 265, 137

Marubashi, K. 1986, Adv. Space Res., 6, 335

McKaig, I. 2001, A\&A, 371, 328

Meegan, C., Lichti, G., Bhat, P. N., et al. 2009, ApJ, 702, 791

Moore, R. L., Sterling, A. C., Hudson, H. S., \& Lemen, J. R. 2001, ApJ, 552, 833

Okamoto, T. J., Tsuneta, S., Lites, B. W., et al. 2008, ApJ, 673, L215

Olmedo, O., \& Zhang, J. 2010, ApJ, 718, 433

Pesnell, W. D., Thompson, B. J., \& Chamberlin, P. C. 2012, Sol. Phys., 275, 3

Priest, E. R., Hood, A. W., \& Anzer, U. 1989, ApJ, 344, 1010

Qiu, J., Hu, Q., Howard, T. A., \& Yurchyshyn, V. B. 2007, ApJ, 659, 758

Rust, D. M., \& Kumar, A. 1994, Sol. Phys., 155, 69

Rust, D., \& Kumar, A. 1996, ApJ, 464, L199

Schou, J., Scherrer, P. H., Bush, R. I., et al. 2012, Sol. Phys., 275, 229

Srivastava, N., \& Venkatakrishnan, P. 2004, J. Geophys. Res. (Space Physics), 109, 10103

Srivastava, A. K., Zaqarashvili, T. V., Kumar, P., \& Khodachenko, M. L. 2010, ApJ, 715, 292

Török, T., \& Kliem, B. 2005, ApJ, 630, L97

Tripathi, D., Kliem, B., Mason, H. E., Young, P. R., \& Green, L. M. 2009, ApJ, 698, L27

van Ballegooijen, A. A., \& Martens, P. C. H. 1989, ApJ, 343, 971

Vourlidas, A. 2014, Plasma Physics and Controlled Fusion, 56, 064001

Vourlidas, A., Lynch, B. J., Howard, R. A., \& Li, Y. 2013, Sol. Phys., 284, 179

Wang, H., Cao, W., Chang, L., et al. 2015, Nature Commun., 6, 7008

Xue, Z., Yan, X., Cheng, X., et al. 2016, Nature Commun., 7, 11837

Yang, S., Zhang, J., \& Xiang, Y. 2015, ApJ, 798, L11

Yurchyshyn, V. B., Wang, H., Goode, P. R., \& Deng, Y. 2001, ApJ, 563, 381

Yurchyshyn, V., Kumar, P., Cho, K.-S., Lim, E.-K., \& Abramenko, V. I. 2015, ApJ, 812, 172

Zhang, J., Cheng, X., \& Ding, M.-D. 2012, Nature Commun., 3, 747 